\title{
Acute chest pain after bench press exercise in a healthy young adult
}

\author{
This article was published in the following Dove Press journal: \\ Open Access Emergency Medicine \\ 22 September 2016 \\ Number of times this article has been viewed
}

\author{
Janet A Smereck' \\ Argyro Papafilippaki² \\ Sawali Sudarshan ${ }^{3}$ \\ 'Department of Emergency \\ Medicine, MedStar Georgetown \\ University Hospital, Washington, DC \\ 2Pennsylvania Hospital, Philadelphia, \\ PA, ${ }^{3}$ Emergency Department, \\ Washington Adventist Hospital, \\ Takoma Park, MD, USA
}

\begin{abstract}
Bench press exercise, which involves repetitive lifting of weights to full arm extension while lying supine on a narrow bench, has been associated with complications ranging in acuity from simple pectoral muscle strain, to aortic and coronary artery dissection. A 39-year-old man, physically fit and previously asymptomatic, presented with acute chest pain following bench press exercise. Diagnostic evaluation led to the discovery of critical multivessel coronary occlusive disease, and subsequently, highly elevated levels of lipoprotein (a). Judicious use of ancillary testing may identify the presence of "high-risk" conditions in a seemingly "low-risk" patient. Emergency department evaluation of the young adult with acute chest pain must take into consideration an extended spectrum of potential etiologies, so as to best guide appropriate management.
\end{abstract}

Keywords: chest pain, coronary artery disease, lipoprotein (a)

\section{Introduction}

Initial evaluation of a patient with chest pain seeks to establish or exclude a serious cause, so as to initiate the appropriate therapeutic pathway. Elements of the history help the clinician ascertain whether a patient with acute chest pain may harbor a lifethreatening disease process. Activities preceding the onset of symptoms may offer clues to the potential etiology. Determining the presence (or absence) of risk factors for cardiovascular disease is of prognostic importance; yet data may be incomplete or lacking at initial emergency department presentation.

\section{Case report}

A 39-year-old man presented with moderately severe 5/10 midsternal chest pain of several hours' duration, after bench press exercise in his home gym. The pain was described as a sensation of a brick weighing on his chest that radiated posteriorly to both shoulders. The pain was not worsened by activity or inspiration. He denied shortness of breath. He also reported tingling paresthesias of both arms, but denied headache, neck pain, or motor weakness. He stated that he felt lightheaded and experienced episodes of sweating following onset of pain; he also reported occasional belching and sensation of acid reflux. He took two aspirin tablets $(325 \mathrm{mg})$ and an over-the-counter liquid antacid, without improvement.

The patient stated that he lifted or pressed weights on a daily basis and ran 6-10 miles each week without prior symptoms of chest pain or dyspnea. He was otherwise in good health; he had no history of hypertension, diabetes, or dyslipid-
Correspondence: Janet A Smereck Med Star Georgetown Emergency Medicine, 3800 Reservoir Road NW, Washington, DC 20007, USA

Email Janet.a.smereck@medstar.net 
emia; he consumed a diet he termed as "healthy"; he took no medications except for multivitamins. He denied recent travel, injury, illness, or immobilization. He denied using tobacco, anabolic steroids, stimulants, or illicit drugs. There was no history of coronary artery disease (CAD) in his parents or siblings.

Physical examination revealed an anxious-appearing man who was thin and muscular. His triage heart rate was 114 beats per minute, blood pressure was $115 / 81$, and respiratory rate 18 . He was afebrile with oxygen saturation of $98 \%$ on room air. Heart rate at the time of physician examination was down to 68 beats per minute. Cardiac monitor revealed sinus rhythm with occasional premature ventricular contractions. His lungs were clear, heart sounds were normal, peripheral pulses symmetric without bruit, abdomen nontender, and peripheral edema absent. There was no calf tenderness. There was mild tenderness of the anterior chest wall, but palpation did not completely reproduce the pain. In addition, there was no spine tenderness; hand grips were equal; and sensation was intact to light touch in the upper extremities.

Electrocardiogram (ECG) on presentation revealed normal sinus rhythm with occasional premature ventricular contractions, right bundle branch block, and subtle ST-segment depressions in lateral leads (Figure 1). There were no prior ECGs available for comparison.

Although the association of the patient's chest pain to bench press exercise and the presence of chest wall tenderness implied a benign cause such as pectoralis muscle strain or costochondritis, the severity of the patient's symptoms prompted investigation for more serious conditions. After return of normal renal function indices by point-of-care testing, he was sent for computerized tomography of the chest with intravenous contrast. The scan was read as normal; heart was not enlarged; and there was no evidence of aortic dissection or aneurysm, pulmonary embolism, or pericardial effusion.
On the patient's return from the radiologic procedure, additional laboratory results became available. Most significantly, troponin-I was elevated at $2.1 \mathrm{ng} / \mathrm{mL}$ (normal, $<0.04 \mathrm{ng} / \mathrm{mL}$ ). Because of continued chest pain, intravenous nitrates were administered; pain reduced slightly to $4 / 10$, but blood pressure fell to $88 / 42$. Normal saline was given in a 1-liter bolus, which raised his blood pressure to 96/53. Cardiology was consulted and the patient was transferred emergently to the catheterization laboratory.

Cardiac catheterization revealed critical multivessel CAD (Figure 2). There was $100 \%$ occlusion of the right coronary artery, $100 \%$ occlusion of the left anterior descending, $90 \%$ occlusion of the circumflex, and 95\% occlusion of the obtuse marginals (one and two) with inferior wall akinesis (Figure 2). There was no evidence of coronary dissection. Hypotension refractory to pressor agents necessitated placement of an intra-aortic balloon pump. As the coronary lesions were extensive, angioplasty with stenting was not technically feasible. The patient underwent emergent four-vessel coronary revascularization. After surgery, cardiac function as determined by echocardiography improved from a pre-

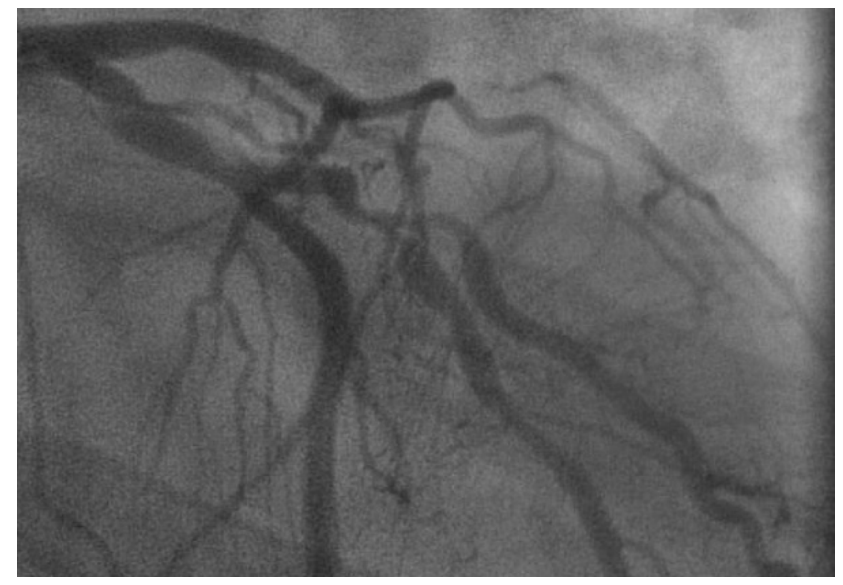

Figure 2 Cardiac catheterization, right anterior oblique projection with 25 degrees of caudal angulation; showing $100 \%$ left anterior descending occlusion, $90 \%$ occlusion of the circumflex, and $95 \%$ occlusion of the obtuse marginals I and 2 .

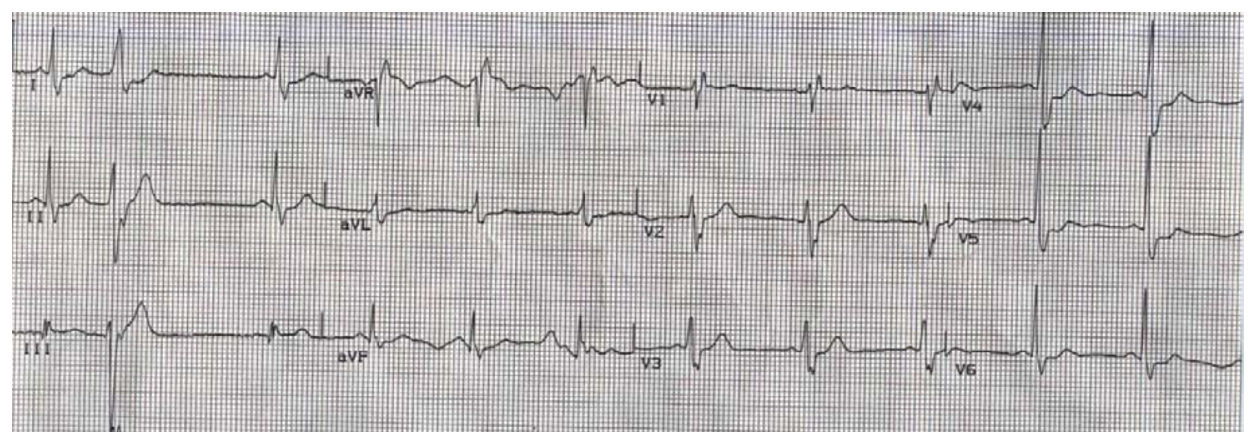

Figure I Electrocardiogram showing normal sinus rhythm and occasional premature ventricular complexes, right bundle branch block, and ST-segment depressions of $0.5-1 \mathrm{~mm}$ in leads $\mathrm{V} 4-\mathrm{V} 6$. 
operative ejection fraction of $30 \%$ up to $40 \%$. Diagnostic investigation of the patient's unexpectedly severe (and previously asymptomatic) coronary occlusive disease included normal cholesterol and triglycerides; however, the patient was found to have highly elevated lipoprotein (a) (Lp [a]) at $251 \mathrm{mg} / \mathrm{dL}$ (normal, $<30 \mathrm{mg} / \mathrm{dL}$ ). Anticardiolipin antibody and antinuclear antigen were negative. As no patient identifiers are used and patient confidentiality is maintained in this single-case report, the Institutional Review Board (IRB) of Georgetown University, as per its IRB Case Reports policy, does not require patient consent for this study.

\section{Discussion}

Clinical evaluation of a healthy adult presenting with acute chest pain must take into consideration an extended spectrum of potential etiologies compared to a patient with known preexisting illness. Less than $10 \%$ of acute myocardial infarctions occur in "young" adults, defined by researchers as age $<40$ to 45 years; however, the incidence appears to be rising over the past several decades..$^{1-3}$ Young adults with myocardial infarction are less likely to have a prior history of angina and more likely to have angiographically normal coronary arteries, or single-vessel disease. ${ }^{4}$ Most young adults with myocardial infarction carry one or more traditional risk factors for CAD as defined by the Framingham Heart Study, including hypertension, diabetes mellitus, tobacco smoking, hyperlipidemia, and family history, defined as a first-degree relative with CAD. ${ }^{3}$

Numerous decision-making instruments exist to assist the emergency physician in risk stratification for evaluation of a patient with potential acute coronary syndrome. ${ }^{5}$ Most clinical decision-making aids combine history, age, and risk factors with ECG findings; cardiac biomarker sampling is firmly imbedded in the decision-making scores. ${ }^{6}$ Although overtesting of cardiac biomarkers carries the potential for false-positive troponin results which then leads to unnecessary follow-up invasive testing, the ubiquitous nature of $\mathrm{CAD}$ and the adverse consequences of missed diagnosis make liberal troponin testing a necessity in the Emergency Department (ED) setting. ${ }^{7}$ The HEART (history, ECG, age, risk factors, troponin) score allows for simple estimation of the risk of major adverse cardiac events in an ED patient with acute chest pain. ${ }^{8}$ High-sensitivity cardiac troponin testing allows for rapid and early detection of acute myocardial injury. ${ }^{9}$ The patient in this case was given a HEART score consistent with intermediate risk of major adverse cardiac events, primarily due to measured troponin elevation $>2$ times the upper limit of normal.

Less commonly recognized etiologies of premature CAD include collagen vascular diseases, Takayasu arteritis,
Table I Uncommon etiologies of coronary artery disease

Collagen vascular diseases, including systemic lupus erythematosus Takayasu arteritis

Kawasaki disease

Antiphospholipid syndrome

Hypereosinophilic syndrome

Thrombocythemia

Hyperlipoproteinemia (a)

Kawasaki disease, antiphospholipid syndrome, hypereosinophilic syndrome, thrombocythemia, and hyperlipoproteinemia (a) (Table 1). ${ }^{10} \mathrm{Lp}$ (a) has prothrombotic and proatherogenic characteristics and structurally resembles plasminogen. ${ }^{11}$ Elevated Lp (a) is genetically mediated and recognized as an independent risk factor for premature CAD. ${ }^{12}$ Levels of $\mathrm{Lp}$ (a) above $60 \mathrm{mg} / \mathrm{dL}$ are positively associated with CAD after adjustment for other cardiovascular risk factors..$^{13}$ There is higher incidence of pathologically elevated Lp (a) in certain population groups, notably South Asians and sub-Saharan Africans. ${ }^{14} \mathrm{Lp}$ (a) abnormalities have been observed to correlate with specific coronary artery plaque morphology in patients with acute myocardial infarction; patients with $\mathrm{Lp}$ (a) levels greater than $25 \mathrm{mg} / \mathrm{dL}$ were observed in one study to have larger plaque core necrosis, thus more prone to plaque rupture. ${ }^{15}$ Optimum treatment of elevated Lp (a) is under investigation; statins, niacin, and fish oil supplements have been prescribed, and apheresis has been utilized in severe, medically refractory cases. ${ }^{16}$ Although intensive exercise results in measurable elevations in acute phase reactants such as fibrinogen, neither positive nor deleterious changes in Lp (a) have been observed following intensive physical activity; moreover, a program of regular exercise has not been shown to decrease hyperlipoproteinemia (a). ${ }^{13,17}$

High levels of physical activity and a program of regular exercise are well understood to confer positive benefits on cardiovascular health. ${ }^{18}$ The risk of acute cardiac events, although increased during a bout of physical activity, is less common in patients who routinely exercise.${ }^{19}$ Clinicians who are involved in exercise preparticipation health screenings are confounded by conflicting guidelines; emphasis is on identifying patients with known disease and concerning symptoms. Routine ECG-guided health assessment is advocated by the European Society of Cardiology; however, the American College of Sports Medicine does not recommend mandatory medical evaluation of asymptomatic athletes given the rare incidence of exercise-related cardiac events in this group. ${ }^{20-22}$

This case emphasizes the fact that apparently healthy and previously asymptomatic young adults can and do have significant CAD. Clinicians are cautioned to be cognizant of uncommon and unrecognized factors that may contribute 
to a patient's coronary risk profile, when evaluating a young adult patient with acute chest pain.

\section{Disclosure}

The authors report no conflicts of interest in this work.

\section{References}

1. Roger VL, Go AS, Lloyd-Jones DM, et al. Heart disease and stroke statistics - 2012 update: a report from the American Heart Association. Circulation. 2012;125(1):e2-e220.

2. Doughty M, Mehta R, Bruckman D, Das S, Karavite D, Tsai T, Eagle K. Acute myocardial infarction in the young - the University of Michigan experience. Am Heart J. 2002;143(1):56-62.

3. Berry JD, Lloyd-Jones DM, Garside DB, Greenland P. Framingham risk score and prediction of coronary heart disease death in young men. $\mathrm{Am}$ Heart J. 2007;154(1):80-86.

4. Trzos E, Uznańska B, Rechciński T, Krzemińska-Pakuła M, Bugała M, Kurpesa M. Myocardial infarction in young people. Cardiol J. 2009; 16(4):307-311.

5. Alley W, Mahler SA. Clinical decision aids for chest pain in the emergency department: identifying low-risk patients. Open Access Emerg Med. 2015;7:85-92.

6. Leite L, Baptista R, Leitao J, et al. Chest pain in the emergency department: risk stratification with Manchester triage system and HEART score. BMC Cardiovasc Disord. 2015;15:48.

7. Makam AN, Nguyen OK. Use of cardiac biomarker testing in the emergency department. JAMA Intern Med. 2015;175(1):67-75.

8. Backus BE, Six AJ, Kelder JC, et al. A prospective validation of the HEART score for chest pain patients at the emergency department. Int J Cardiol. 2013;168(3):2153-2158.

9. Mueller C, Giannitsis E, Christ M, et al. Multicenter evaluation of a 0-hour/1-hour algorithm in the diagnosis of myocardial infarction with high-sensitivity cardiac troponin T. Ann Emerg Med. 2016;68(1): 76-87.e4.

10. Gambhir JK, Kaur H, Gambhir DS, Prabhu KM. Lipoprotein(a) as an independent risk factor for coronary artery disease in patients below 40 years of age. Indian Heart J. 2000;52(4):411-415.
11. Boffa MB, Koschinsky ML. Lipoprotein (a): truly a direct prothrombotic factor in cardiovascular disease? J Lipid Res. 2016;57(5):745-757.

12. Anderson JL, Knight S, May HT, et al. Validation and quantification of genetic determinants of lipoprotein-a levels and predictive value for angiographic coronary artery disease. Am J Cardiol. 2013;112(6):799-804.

13. Berthold HK, Gouni-Berthold I. Hyperlipoproteinemia(a): clinical significance and treatment options. Atheroscler Suppl. 2013;14(1):1-5.

14. Bilen O, Kamal A, Virani SS. Lipoprotein abnormalities in South Asians and its association with cardiovascular disease: current state and future directions. World J Cardiol. 2016;8(3):247-257.

15. Hikita H, Shigeta T, Kojima K, et al. Lipoprotein(a) is an important factor to determine coronary artery plaque morphology in patients with acute myocardial infarction. Coron Artery Dis. 2013;24(5):381-385.

16. Waldmann E, Parhofer KG. Lipoprotein apheresis to treat elevated lipoprotein(a). J Lipid Res. Epub 2016 Feb 17.

17. Byrne DJ, Jagroop IA, Montgomery HE, Thomas M, Mikhailidis DP, Milton NG, Winder AF. Lipoprotein (a) does not participate in the early acute phase response to training or extreme physical activity and is unlikely to enhance any associated immediate cardiovascular risk. $J$ Clin Pathol. 2002;55(4):280-285.

18. Lavie CJ, Arena R, Swift DL, et al. Exercise and the cardiovascular system: clinical science and cardiovascular outcomes. Circ Res. 2015;117(2):207-219.

19. Whitfield GP, Pettee Gabriel KK, Rahbar MH, Kohl HW. Application of the American Heart Association/American College of Sports Medicine Adult Participation Screening Checklist to a nationally representative sample of US adults aged 40 years from the National Health and Nutrition Examination Survey 2001-2004. Circulation. 2014;129(10):1113-1120.

20. Corrado D, Pelliccia A, Bjørnstad HH, et al. Cardiovascular preparticipation screening of young competitive athletes for prevention of sudden death: proposal for a common European protocol. Consensus Statement of the Study Group of Sport Cardiology of the Working Group of Cardiac Rehabilitation and Exercise Physiology and the Working Group of Myocardial and Pericardial Diseases of the European Society of Cardiology. Eur Heart J. 2005;26(5):516-524.

21. Roberts WO, Asplund CA, O’Connor FG, Stovitz SD. Cardiac preparticipation screening for the young athlete: why the routine use of ECG is not necessary. J Electrocardiol. 2015;48(3):311-315.

22. Marijon E, Tafflet M, Celermajer DS, et al. Sports-related sudden death in the general population. Circulation. 2011;124(6):672-681.
Open Access Emergency Medicine

\section{Publish your work in this journal}

The Open Access Emergency Medicine is an international, peerreviewed, open access journal publishing original research, reports, editorials, reviews and commentaries on all aspects of emergency medicine. The manuscript management system is completely online and includes a very quick and fair peer-review system, which is all

\section{Dovepress}

easy to use. Visit http://www.dovepress.com/testimonials.php to read real quotes from published authors. 